\title{
The initial involution patterns of permutations
}

\author{
Dongsu Kim* \\ Department of Mathematics \\ Korea Advanced Institute of Science and Technology \\ Daejeon 305-701, Korea \\ dskim@math.kaist.ac.kr \\ and \\ Jang Soo Kim \\ Department of Mathematics \\ Korea Advanced Institute of Science and Technology \\ Daejeon 305-701, Korea \\ jskim@kaist.ac.kr
}

Submitted: July 18, 2006; Accepted: Dec 10, 2006; Published: Jan 3, 2007

Mathematics Subject Classification: 05A05, 05A15

\begin{abstract}
For a permutation $\pi=\pi_{1} \pi_{2} \cdots \pi_{n} \in S_{n}$ and a positive integer $i \leq n$, we can view $\pi_{1} \pi_{2} \cdots \pi_{i}$ as an element of $S_{i}$ by order-preserving relabeling. The $j$-set of $\pi$ is the set of $i$ 's such that $\pi_{1} \pi_{2} \cdots \pi_{i}$ is an involution in $S_{i}$. We prove a characterization theorem for $j$-sets, give a generating function for the number of different $j$-sets of permutations in $S_{n}$. We also compute the numbers of permutations in $S_{n}$ with a given $j$-set and prove some properties of them.
\end{abstract}

\section{Introduction}

In order to count standard Young tableaux containing a given tableau, McKay, Morse and Wilf [2] considered the number of involutions in $S_{n}$ containing a given permutation $\sigma$, and Jaggard [1] found a formula for the number, showing that the number depends only on the ' $j$-set' of $\sigma$.

*The first author was partially supported by KRF grant R05-2004-000-11511-0. 
Let $S_{n}$ denote the set of all permutations of $[n]=\{1,2, \ldots, n\}$. A permutation $\pi \in S_{n}$ is called an involution, if $\pi=\pi^{-1}$. Let $w=w_{1} w_{2} \cdots w_{j}$ be a sequence of $j$ distinct integers. The pattern of $w$ is the permutation $\sigma=\sigma_{1} \sigma_{2} \cdots \sigma_{j} \in S_{j}$, satisfying $\sigma_{r}<\sigma_{s}$ if and only if $w_{r}<w_{s}$. For a permutation $\pi=\pi_{1} \pi_{2} \cdots \pi_{n} \in S_{n}$, the pattern of $\pi_{1} \pi_{2} \cdots \pi_{i}$ is called the initial $i$-pattern of $\pi$.

Definition 1.1. The $j$-set of a permutation $\pi \in S_{n}$ with $n \geq 2$, denoted by $J(\pi)$, is the set of all nonnegative integers $i$ such that the initial $i$-pattern of $\pi$ is an involution. For convenience, we regard the initial 0 -pattern as an involution.

Jaggard in [1, Proposition 3.4] uses $j$-sets to classify permutations according to subsequence containment by involutions. He gives data on $j$-sets and proves several properties, proposing some questions regarding the $j$-sets. One of the questions is about the number of different sets which can be $j$-sets of permutations in $S_{n}$. We answer the question with a characterization theorem for $j$-sets and, moreover, find an explicit generating function for the numbers.

Definition 1.2. For a permutation $\pi \in S_{n}$, let $M(\pi)$ denote the permutation matrix corresponding to $\pi$, that is, the $(i, j)$-entry of $M(\pi)$ is 1 if $\pi(i)=j$; and 0 , otherwise. For subsets $A, B$ of $[n]$, let $M(\pi ; A, B)$ be the submatrix of $M(\pi)$ with row set $A$ and column set $B$. For $k \in[n]$, let $M_{\text {row }}(\pi ; k)=M(\pi ;[k], \pi([k]))$, where $\pi(A)$ denotes $\{\pi(i): i \in A\}$. Similarly, $M_{\text {col }}(\pi ; k)=M\left(\pi ; \pi^{-1}([k]),[k]\right)$. Finally, $M(\pi ; k)=M\left(\pi ;[k] \cap \pi^{-1}([k]),[k] \cap\right.$ $\pi([k]))$. The matrix $M(\pi ; k)$ is allowed to be the empty matrix, the matrix with no rows and columns. For a technical reason we call the empty matrix symmetric.

According to the above definition, $M_{\text {row }}(\pi ; k)$ is obtained from $M(\pi)$ by removing the last $n-k$ rows and then deleting columns consisting of only zeroes. $M(\pi ; k)$ is obtained from $M(\pi)$ by cropping the first $k$ rows and $k$ columns, and then deleting zero rows and columns. Note that for any permutation $\pi \in S_{n}, M\left(\pi^{-1}\right)=M(\pi)^{T}$, the transpose of $M(\pi)$, and $M_{\text {row }}(\pi ; k)=M_{\text {col }}\left(\pi^{-1} ; k\right)^{T}$.

For example, if $\pi=541263 \in S_{6}$ then $M(\pi)=\left(\begin{array}{cccccc}0 & 0 & 0 & 0 & 1 & 0 \\ 0 & 0 & 0 & 1 & 0 & 0 \\ 1 & 0 & 0 & 0 & 0 & 0 \\ 0 & 1 & 0 & 0 & 0 & 0 \\ 0 & 0 & 0 & 0 & 0 & 1 \\ 0 & 0 & 1 & 0 & 0 & 0\end{array}\right)$,

$M_{\text {row }}(\pi ; 4)=\left(\begin{array}{cccc}0 & 0 & 0 & 1 \\ 0 & 0 & 1 & 0 \\ 1 & 0 & 0 & 0 \\ 0 & 1 & 0 & 0\end{array}\right), M_{\text {col }}(\pi ; 4)=\left(\begin{array}{cccc}0 & 0 & 0 & 1 \\ 1 & 0 & 0 & 0 \\ 0 & 1 & 0 & 0 \\ 0 & 0 & 1 & 0\end{array}\right), M(\pi ; 4)=\left(\begin{array}{ccc}0 & 0 & 1 \\ 1 & 0 & 0 \\ 0 & 1 & 0\end{array}\right)$

and $M(\pi ; 2)$ is the empty matrix.

If $\sigma$ is the initial $k$-pattern of $\pi \in S_{n}$, then $M(\sigma)=M_{\text {row }}(\pi ; k)$. Since $\pi \in S_{n}$ is an involution if and only if $M(\pi)$ is symmetric, we have $k \in J(\pi)$ if and only if $M_{\text {row }}(\pi ; k)$ is symmetric.

Let $\pi \in S_{n}$ be an involution, i.e. $\pi=\pi^{-1}$. Then $M(\pi)$ is symmetric and $M(\pi ; k)$ is symmetric for all $k=1,2, \ldots, n$. Since $M_{\text {row }}(\pi ; k)=M_{\text {col }}\left(\pi^{-1} ; k\right)^{T}=M_{\text {col }}(\pi ; k)^{T}$, 
$M_{\text {row }}(\pi ; k)$ is symmetric if and only if $M_{\text {col }}(\pi ; k)$ is symmetric, equivalently $M_{\text {row }}(\pi ; k)=$ $M_{c o l}(\pi ; k)$. In summary, we get the following proposition.

Proposition 1.3. For any involution $\pi \in S_{n}$, the following are equivalent.

1. $k \in J(\pi)$.

2. $M_{\text {row }}(\pi ; k)$ is symmetric.

3. $M_{c o l}(\pi ; k)$ is symmetric.

4. $M_{\text {row }}(\pi ; k)=M_{\text {col }}(\pi ; k)$.

The rest of the paper is organized as follows. We present in $\S 2$ the main result involving a criterion of $j$-sets, find a generating function of the number of $j$-sets in $\S 3$, consider the number of $\pi \in S_{n}$ with $J(\pi)=J$, denoted by $p_{n}(J)$, in $\S 4$. We also give a recurrence relation for the numbers $p_{n}(J)$ and prove a divisibility property of them.

\section{Criterion Theorem for $j$-sets}

For $n>1$ and $\pi \in S_{n}$, we always have $\{0,1,2\} \subset J(\pi) \subset\{0,1,2, \ldots, n\}$. Let $\pi$ be a permutation and $\sigma$ be the initial $k$-pattern of $\pi$. For integer $i \leq k$, the initial $i$-pattern of $\pi$ is equal to the initial $i$-pattern of $\sigma$. Thus $J(\sigma)=J(\pi) \cap[k]$. So $J=\left\{a_{1}, a_{2}, \ldots, a_{k}\right\}$, where $a_{1}<a_{2}<\cdots<a_{k}$, is a $j$-set if and only if $\left\{a_{1}, a_{2}, \ldots, a_{i}\right\}$ is a $j$-set for all $i \leq k$. To classify all $j$-sets, it is sufficient to determine when $J \cup\{n\}$ is a $j$-set, for a $j$-set $J$ and an integer $n$ greater than $\max (J)$. The lemma below shows that if $J$ is a $j$-set then there is an involution $\pi \in S_{m}$ satisfying $J(\pi)=J$ where $m=\max (J)$.

Lemma 2.1. Let $J$ be a $j$-set with $\max (J)=m \geq 2$. Then for any integer $n \geq m$, there is a permutation $\pi \in S_{n}$ with $J(\pi)=J$.

Proof. Since $J$ is a $j$-set, there is a permutation $\sigma \in S_{l}$ for some integer $l \geq m$ such that $J(\sigma)=J$. For any $n$ with $m \leq n \leq l$, the initial $n$-pattern of $\sigma$ is a permutation in $S_{n}$ with $j$-set $J$. So it remains to show that for any $n>l$, there exists $\pi \in S_{n}$ with $J(\pi)=J$. This can be established by an inductive argument, if it can be shown just for $n=l+1$.

There are $l+1$ permutations $\tau_{1}, \tau_{2}, \ldots, \tau_{l+1}$ in $S_{l+1}$ with $\tau_{i}(l+1)=i$ whose initial $l$ patterns are $\sigma$. For each $i$ the $j$-set of $\tau_{i}$ is either $J$ or $J \cup\{l+1\}$. In fact, $J\left(\tau_{i}\right)=J \cup\{l+1\}$ if and only if $\tau_{i}$ itself is an involution. If $\tau_{i}$ is an involution for $i \leq l$, then $\tau_{i}(i)=l+1$, which implies $\sigma(i)=l$, equivalently, $i=\sigma^{-1}(l)$. Since $l \geq 2$, we can take $\pi=\tau_{j}$ with $j \neq i$ to force $J(\pi)=J$.

For convenience, we define $I_{k}$ to be the permutation matrix of $12 \cdots k \in S_{k}$ and $I_{k}^{\prime}$ the permutation matrix of $k(k-1) \cdots 1 \in S_{k} . I_{k}$ is the identity matrix and $I_{k}^{\prime}=\left(a_{i j}\right)$ with $a_{i j}=1$, if $i+j=k+1 ; 0$, otherwise. 
Lemma 2.2. Let $M$ be an $n \times n$ permutation matrix of the form

$$
M=\left(\begin{array}{c}
A \\
B
\end{array}\right)
$$

where $A$ is an $(n-k) \times n$ matrix and $B$ a $k \times n$ matrix. Let $s$ be a positive integer and

$$
N=\left(\begin{array}{cc}
A & 0 \\
0 & I_{s}^{\prime} \\
B & 0
\end{array}\right)
$$

If both $M$ and $N$ are symmetric, then $M$ is of the form

$$
M=\left(\begin{array}{cc}
C & 0 \\
0 & I_{k}^{\prime}
\end{array}\right)
$$

where $C$ is an appropriate $(n-k) \times(n-k)$ symmetric matrix.

Proof. For $1 \leq i \leq k+s$, let $e_{i}$ be the $(n+s+1-i, n-k+i)$-entry of $N$. The positions of $e_{i}$ 's are shown below in $N$ and $M$, omitting all other entries.

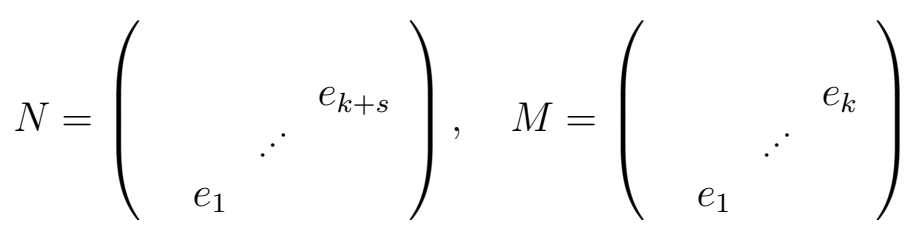

It is sufficient to show that $e_{i}=1$ for all $i, 1 \leq i \leq k$.

Since $N$ is symmetric, $e_{i}=e_{k+s+1-i}$ for $1 \leq i \leq k+s$, and since $M$ is also symmetric, $e_{i}=e_{k+1-i}$ for $1 \leq i \leq k$. From the definition of $N$ we can read $e_{k+1}=\cdots=e_{k+s}=1$, which imply $e_{1}=\cdots=e_{s}=1$. Now if $e_{i}=1$ and $i+s \leq k$ then $e_{i+s}=1$ because

$$
e_{i+s}=e_{k+s+1-(i+s)}=e_{k+1-i}=e_{i}=1 .
$$

Thus $e_{i}=1$ for all $i \leq k$.

The above lemma is actually about two permutation matrices corresponding to involutions. It can be phrased in terms of permutations, but matrix version is easier to handle.

Corollary 2.3. Let $\pi \in S_{n}$ and $\pi(n)=n-k+1$. Assume $n-1, n \in J(\pi)$. Then

$$
M(\pi)=\left(\begin{array}{cc}
C & 0 \\
0 & I_{k}^{\prime}
\end{array}\right)
$$

for some $(n-k) \times(n-k)$ symmetric matrix $C$. Moreover, $n-k, n-k+1, \ldots, n \in J(\pi)$. 
Proof. If $k=1$, it is obvious. Assume $k>1$. Since $\pi(n-k+1)=n$,

$$
M(\pi)=\left(\begin{array}{cc}
A & 0 \\
0 & 1 \\
B & 0
\end{array}\right)
$$

where $A$ and $B$ are appropriate matrices of size $(n-k) \times(n-1)$ and $(k-1) \times(n-1)$ respectively. Since $n \in J(\pi), M(\pi)$ is symmetric, and $n-1 \in J$ implies the matrix $\left(\begin{array}{c}A \\ B\end{array}\right)=M_{c o l}(\pi ; n-1)$ is symmetric. Now apply Lemma 2.2 to matrices $\left(\begin{array}{c}A \\ B\end{array}\right)$ and $M(\pi)$ to complete the proof.

Let $J$ be a $j$-set with $\max (J)=m$. By Lemma 2.1, there is a permutation $\pi \in S_{m}$ such that $J(\pi)=J$. Since $\pi=\pi_{1} \pi_{2} \cdots \pi_{m}$ is an involution, $\pi^{\prime}=\pi_{1} \pi_{2} \cdots \pi_{m}(m+1) \in S_{m+1}$ is also an involution. Thus $J \cup\{m+1\}$ is always a $j$-set. So we are interested in determining when $J \cup\{n\}$ with $n \geq m+2$ is a $j$-set.

Theorem 2.4. Let $J$ be a $j$-set with $\max (J)=m \geq 2$ and $n \geq m+2$. Then $J \cup\{n\}$ is $a j$-set if and only if $n-m \geq m-\max (J \cap[m-2])$.

Proof. ( $\Longrightarrow)$ Take a permutation $\pi \in S_{n}$ such that $J(\pi)=J \cup\{n\}$, the existence of which is guaranteed by Lemma 2.1. Let $\sigma \in S_{m}$ be the initial $m$-pattern of $\pi$. Then both $\pi$ and $\sigma$ are involutions and $J(\sigma)=J$. We partition $M(\pi)$ into four blocks as follows,

$$
M(\pi)=\begin{aligned}
& m \\
& n-m-m \\
& n
\end{aligned}\left(\begin{array}{cc}
A & B \\
B^{T} & C
\end{array}\right),
$$

where $A$ is of size $m \times m, B$ is of size $m \times(n-m)$ and $C$ is of size $(n-m) \times(n-m)$. The sizes of blocks are shown on the margins of the matrix.

Let $s$ denote the number of 1's in $B$. Then $0 \leq s \leq n-m$.

We first show that $m-s \in J . M(\pi ; m)$, which is obtained from $M(\pi)$, is also obtained from $A$ by deleting zero rows and columns, and $M(\sigma)=M_{\text {row }}(\pi ; m)$ is the matrix obtained from $(A B)$ by deleting zero columns. So we have $M(\pi ; m)=M_{\text {col }}(\sigma ; m-s)$. This implies that $M_{\text {col }}(\sigma ; m-s)$ is symmetric, since $M(\pi ; m)$ is symmetric. By Proposition 1.3, $m-s \in J(\sigma)=J$.

When $s \geq 2$, we clearly have $m-\max (J \cap[m-2]) \leq m-(m-s)=s \leq n-m$.

Suppose $s=0$. Then 1 appears in neither $B$ nor $B^{T}$, so

$$
M(\pi)=\left(\begin{array}{cc}
A & 0 \\
0 & C
\end{array}\right) .
$$

Since $A$ is symmetric, we have $m+1 \in J(\pi)$, which implies $J(\pi) \neq J \cup\{n\}$, contradicting the choice of $\pi$. Thus $s$ can not be zero.

Now suppose $s=1$, i.e. $B$ has only one 1 . Since $m-s \in J(\sigma)$, we have $m-1, m \in$ $J(\sigma)$. Let $k$ be the positive integer satisfying $\sigma(m)=m-k+1$. From Corollary 2.3, we 
have $m-k, m-k+1, \ldots, m \in J$. If $k \geq 2$ then $m-2 \in J$ and $m-\max (J \cap[m-2])=$ $2 \leq n-m$. If $k=1$, then

$$
M(\sigma)=\left(\begin{array}{cc}
M(\pi ; m) & 0 \\
0 & 1
\end{array}\right) .
$$

If 1 is not in the first row of $B^{T}$, then

$$
M_{\text {row }}(\pi ; m+1)=\left(\begin{array}{cc}
M(\pi ; m) & 0 \\
0 & D
\end{array}\right),
$$

where $D=\left(\begin{array}{ll}1 & 0 \\ 0 & 1\end{array}\right)$ or $\left(\begin{array}{ll}0 & 1 \\ 1 & 0\end{array}\right)$, both of which are symmetric. So we get $m+1 \in J(\pi)$, which contradicts the assumption $J(\pi)=J \cup\{n\}$. If 1 is in the first row of $B^{T}$, then $M_{\text {row }}(\pi ; m+1)=M(\pi ; m+1)$ because there is no 1 in $B$ except in the first column. Thus $M_{\text {row }}(\pi ; m+1)$ is symmetric, and $m+1 \in J(\pi)$, which implies $J(\pi) \neq J \cup\{n\}$, contradicting the choice of $\pi$.

$(\Longleftarrow)$ We will show this by constructing $\pi \in S_{n}$ satisfying the condition $J(\pi)=J \cup\{n\}$.

Let $\sigma \in S_{m}$ be an involution with $J(\sigma)=J$.

We may assume that for any $e \geq 2$ and any $(m-e) \times(m-e)$ symmetric matrix $Z$,

$$
M(\sigma) \neq\left(\begin{array}{cc}
Z & 0 \\
0 & I_{e}^{\prime}
\end{array}\right) .
$$

Suppose $M(\sigma)=\left(\begin{array}{cc}Z & 0 \\ 0 & I_{e}^{\prime}\end{array}\right)$ for some $e \geq 2$ and an $(m-e) \times(m-e)$ symmetric matrix $Z$. Then $m-e, m-e+1, \ldots, m \in J(\sigma)$. Define $\sigma^{\prime} \in S_{m}$ by the relation $M\left(\sigma^{\prime}\right)=\left(\begin{array}{cc}Z & 0 \\ 0 & I_{e}\end{array}\right)$, which implies $J\left(\sigma^{\prime}\right)=J(\sigma)$. Since we can replace $\sigma$ with $\sigma^{\prime}$, if necessary, we may assume (1).

Let $k=n-m$ and $r=m-\max (J \cap[m-2])$. If $J=\{0,1,2\}$, then $m-r$ is zero and the matrices below with $m-r$ rows or columns are empty matrices. Partition $M(\sigma)$ into $\left(\begin{array}{c}A \\ B\end{array}\right)$, where $A$ is an $(m-r) \times m$ matrix and $B$ is an $r \times m$ matrix. Since $\sigma$ is an involution, $M(\sigma)=\left(A^{T} B^{T}\right)$. Let $W$ be the set of indices of columns of $A$ containing 1 . Then $i \in W$ if and only if the $i$-th row of $A^{T}$ contains 1 . Let $A_{0}$ be the $m \times m$ matrix whose $W \times W$ submatrix is $M_{\text {row }}(\sigma ; m-r)$ and whose entries outside the submatrix are 0 . Since $M_{\text {row }}(\sigma ; m-r)$ is symmetric, so is $A_{0}$.

Let $\pi \in S_{n}$ be the involution whose corresponding matrix is

$$
M(\pi)=\left(\begin{array}{ccc}
A_{0} & 0 & B^{T} \\
0 & I_{k-r}^{\prime} & 0 \\
B & 0 & 0
\end{array}\right) .
$$

The matrix in (2) is a symmetric permutation matrix and $M_{\text {row }}(\pi ; m)=M(\sigma)$.

We claim $J(\pi)=J \cup\{n\}$. 
Since $\pi$ is an involution and $\sigma$ is the initial $m$-pattern of $\pi$, it is enough to show that $m+i \notin J(\pi)$ for $i=1, \ldots, k-1$. If $m+i \in J(\pi)$ for some $i=1, \ldots, k-r$, then

$$
M_{c o l}(\pi ; m+i)=\left(\begin{array}{cc}
A & 0 \\
0 & I_{i}^{\prime} \\
B & 0
\end{array}\right)
$$

is symmetric. Thus, by Lemma 2.2,

$$
M(\sigma)=\left(\begin{array}{cc}
M_{\text {row }}(\sigma ; m-r) & 0 \\
0 & I_{r}^{\prime}
\end{array}\right)
$$

which contradicts the assumption (1). Thus $m+i \notin J(\pi)$ for $i=1, \ldots, k-r$.

Suppose $m+(k-r)+l \in J(\pi)$ for some $l, 1 \leq l<r$.

Since $M_{c o l}(\pi ; m+k-r+l)$ is symmetric, when we remove the last $l$ columns and rows from $M_{\text {col }}(\pi ; m+k-r+l)$ and delete zero rows and columns, we get the following symmetric matrix $D$.

$$
D=\begin{aligned}
& m-l \\
& k-r \\
& r-l
\end{aligned}\left(\begin{array}{cc}
A^{\prime} & 0 \\
0 & I_{k-r}^{\prime} \\
C & 0
\end{array}\right),
$$

where $A^{\prime}$ and $C$ are the $(m-r) \times(m-l)$ and $(r-l) \times(m-l)$ matrices respectively satisfying $M_{\text {row }}(\sigma ; m-l)=\left(\begin{array}{c}A^{\prime} \\ C\end{array}\right)$. Note that $I_{k-r}^{\prime}$ survives in $D$ since the 1 's in the last $l$ columns of $M_{\text {col }}(\pi ; m+k-r+l)$ come from $B^{T}$ in $M(\pi)$.

We consider two cases separately.

CASE $1: r-l>k-r$

When we remove the last $k-r$ columns and rows of the matrix $D$ and delete zero rows and columns, we get a symmetric matrix $M_{\text {row }}(\sigma ; m-(l+k-r))$. So $m-(l+k-r) \in J$. Since $r-l>k-r$, i.e. $r>l+k-r$, we deduce $\max (J \cap[m-2])=m-r<m-(l+k-r)<m$, which implies $l+k-r=1$. Because $l \geq 1$ and $k-r \geq 0$, we obtain $l=1$ and $k=r$. Thus $M(\pi)$ in $(2)$ reduces to

$$
M(\pi)=\left(\begin{array}{cc}
A_{0} & B^{T} \\
B & 0
\end{array}\right) .
$$

The supposition $m+(k-r)+l \in J(\pi)$ above and $l+k-r=1$ imply $m+1 \in J(\pi)$. Since $m, m+1 \in J(\pi)$, by Corollary 2.3, $M_{\text {col }}(\pi ; m+1)=\left(\begin{array}{cc}X & 0 \\ 0 & I_{s}^{\prime}\end{array}\right)$ for some $s$. Moreover, we have $s>r$ because in (2) the last $r$ entries are 0 in the $(m+1)$-th column of $M(\pi)$ and no row of $B$ is deleted while obtaining $M_{c o l}(\pi ; m+1)$ from $M(\pi)$. Thus $M(\sigma)=$ $M_{c o l}(\pi ; m)=\left(\begin{array}{cc}X & 0 \\ 0 & I_{s-1}^{\prime}\end{array}\right)$ with $s-1 \geq r \geq 2$, which contradicts our assumption (1). 
CASE $2: r-l \leq k-r$

Let $E$ be the submatrix of $D$ with the last $k-l$ rows and columns. Then $E$ is symmetric and $E=\left(\begin{array}{cc}0 & I_{k-r}^{\prime} \\ Y & 0\end{array}\right)$ for an appropriate matrix $Y$. Because $k-r \geq(k-l) / 2$ and $E$ is symmetric, $E=I_{k-l}^{\prime}$. Then we have

$$
D=\left(\begin{array}{cc}
M_{\text {row }}(\sigma ; m-r) & 0 \\
0 & I_{k-l}^{\prime}
\end{array}\right)
$$

Thus

$$
M_{\text {row }}(\sigma ; m-l)=\left(\begin{array}{cc}
M_{\text {row }}(\sigma ; m-r) & 0 \\
0 & I_{r-l}^{\prime}
\end{array}\right),
$$

which implies $m-r, m-r+1, \ldots, m-l \in J$. Since $m-r=\max (J \cap[m-2])$ and $1 \leq l<r$, we conclude $r=2$ and $l=1$. Then $n-1=m+k-r+l \in J(\pi)$. Since $n-1, n \in J(\pi)$, by Corollary 2.3, for an appropriate matrix $G$ and an integer $t$, $M(\pi)=\left(\begin{array}{cc}G & 0 \\ 0 & I_{t}^{\prime}\end{array}\right)$ and comparing with $(2)$, we get $M(\pi)=\left(\begin{array}{cc}M_{\text {row }}(\sigma ; m-2) & 0 \\ 0 & I_{k+2}^{\prime}\end{array}\right)$ and $M(\sigma)=\left(\begin{array}{cc}M_{\text {row }}(\sigma ; m-2) & 0 \\ 0 & I_{2}^{\prime}\end{array}\right)$, contradicting the assumption (1).

\section{Permutation version of the construction.}

We can describe the above construction in terms of permutation itself without resorting to its matrix representation. This description is simpler and allows us to grasp the idea behind the construction but the matrix version has advantage in a rigorous proof.

Let $J$ be the $j$-set in the above proof and $\sigma, \pi$ denote the permutations there. Recall $\max (J)=m, J(\sigma)=J$. Let $k=n-m$ and assume $k \geq r=m-\max (J \cap[m-2])$. Then the permutation $\pi=\pi_{1} \pi_{2} \cdots \pi_{n} \in S_{n}$ is defined by

$$
\pi_{j}= \begin{cases}\tau_{j}, & 1 \leq j \leq m, \\ m+n+1-r-j, & m+1 \leq j \leq n-r, \\ \sigma_{j-n+m}, & n-r+1 \leq j \leq n,\end{cases}
$$

where $\tau=\tau_{1} \tau_{2} \cdots \tau_{m}$ is the permutation of the set

$$
[n] \backslash\left(\{m+1, m+2, \ldots, m+k-r\} \cup\left\{\sigma_{m-r+1}, \sigma_{m-r+2}, \ldots, \sigma_{m}\right\}\right),
$$

whose pattern is $\sigma$. That is,

$$
\begin{aligned}
\pi & =\pi_{1} \pi_{2} \cdots \pi_{m} \pi_{m+1} \pi_{m+2} \cdots \pi_{m+k-r} \pi_{n-r+1} \cdots \pi_{n} \\
& =\tau_{1} \tau_{2} \cdots \tau_{m}(m+k-r)(m+k-r-1) \cdots(m+1) \sigma_{m-r+1} \cdots \sigma_{m}
\end{aligned}
$$

Example 2.5. Let $\sigma=163542$. Then $J(\sigma)=\{0,1,2,3,6\}$. We will construct $\pi \in S_{12}$ with $J(\pi)=\{0,1,2,3,6,12\}$. In this case $(r, k)=(3,6)$. Since $k \geq r$ we can construct $\pi$. The last 3 elements of $\pi$ are the last 3 elements of $\sigma$, i.e. 542 . The middle part of $\pi$ is 
987 . The first part of $\pi$ is the permutation of $\{1,3,6,10,11,12\}$ whose pattern is $\sigma$, that is, 112611103 . Thus $\pi=112611103987542$.

The corresponding $M(\sigma)$ and $M(\pi)$ in $(2)$ are

$$
M(\sigma)=\left(\begin{array}{cccccc}
1 & 0 & 0 & 0 & 0 & 0 \\
0 & 0 & 0 & 0 & 0 & 1 \\
0 & 0 & 1 & 0 & 0 & 0 \\
0 & 0 & 0 & 0 & 1 & 0 \\
0 & 0 & 0 & 1 & 0 & 0 \\
0 & 1 & 0 & 0 & 0 & 0
\end{array}\right), M(\pi)=\left(\begin{array}{cccccc|ccc|ccc}
1 & 0 & 0 & 0 & 0 & 0 & 0 & 0 & 0 & 0 & 0 & 0 \\
0 & 0 & 0 & 0 & 0 & 0 & 0 & 0 & 0 & 0 & 0 & 1 \\
0 & 0 & 0 & 0 & 0 & 1 & 0 & 0 & 0 & 0 & 0 & 0 \\
0 & 0 & 0 & 0 & 0 & 0 & 0 & 0 & 0 & 0 & 1 & 0 \\
0 & 0 & 0 & 0 & 0 & 0 & 0 & 0 & 0 & 1 & 0 & 0 \\
0 & 0 & 1 & 0 & 0 & 0 & 0 & 0 & 0 & 0 & 0 & 0 \\
\hline 0 & 0 & 0 & 0 & 0 & 0 & 0 & 0 & 1 & 0 & 0 & 0 \\
0 & 0 & 0 & 0 & 0 & 0 & 0 & 1 & 0 & 0 & 0 & 0 \\
0 & 0 & 0 & 0 & 0 & 0 & 1 & 0 & 0 & 0 & 0 & 0 \\
\hline 0 & 0 & 0 & 0 & 1 & 0 & 0 & 0 & 0 & 0 & 0 & 0 \\
0 & 0 & 0 & 1 & 0 & 0 & 0 & 0 & 0 & 0 & 0 & 0 \\
0 & 1 & 0 & 0 & 0 & 0 & 0 & 0 & 0 & 0 & 0 & 0
\end{array}\right) .
$$

\section{Generating Function of the number of $j$-sets}

Let $\mathcal{J}$ be the set of all $j$-sets. Recall that each member of $\mathcal{J}$ contains $\{0,1,2\}$. Let $\mathcal{J}_{n}$ be the set of $j$-sets of permutations in $S_{n}$ for $n \geq 2$. Recall that a set $J$ is called a $j$-set if there is a permutation $\pi$ satisfying $J(\pi)=J$.

Define $F(x, y, z)$ and $G(u, z)$ as follows:

$$
\begin{gathered}
F(x, y, z)=\sum_{n \geq 2} \sum_{J \in \mathcal{J}_{n}} x^{n} y^{\max (J)} z^{|J|}=\sum_{n, m, l \geq 2} f(n, m, l) x^{n} y^{m} z^{l}, \\
G(u, z)=\sum_{J \in \mathcal{J}} u^{\max (J)} z^{|J|}=\sum_{m, l \geq 2} g(m, l) u^{m} z^{l} .
\end{gathered}
$$

So $f(n, m, l)$ is the number of $J \in \mathcal{J}_{n}$ with $\max (J)=m$ and $|J|=l$, and $g(m, l)$ is the number of $J \in \mathcal{J}$ with $\max (J)=m$ and $|J|=l$. Lemma 2.1 says that if $J \in \mathcal{J}$ then $J \in \mathcal{J}_{n}$ for all $n \geq \max (J)$. Thus $\mathcal{J}_{n}$ is the set of all $j$-sets whose maximal elements are less than or equal to $n$, and consequently, $f(n, m, l)=g(m, l)$ for $n \geq m$. So we obtain

$$
F(x, y, z)=\frac{G(x y, z)}{1-x} .
$$

\section{Inductive definition of $j$-sets.}

Theorem 2.4 and the comment preceding it give a criterion for $j$-sets, which can be used to determine $j$-sets inductively as in the following corollary.

Corollary 3.1. Assume that $\left\{a_{1}, \ldots, a_{r-1}\right\}$ is a $j$-set and $a_{1}<a_{2}<\cdots<a_{r}$. Then $\left\{a_{1}, \ldots, a_{r}\right\}$ is a $j$-set if and only if one of the following holds:

1. $a_{r}-a_{r-1}=1$ 
2. $a_{r-1}-a_{r-2} \neq 1$ and $a_{r}-a_{r-1} \geq a_{r-1}-a_{r-2}$

3. $a_{r-1}-a_{r-2}=1$ and $a_{r}-a_{r-1} \geq a_{r-1}-a_{r-3}$

We illustrate how to use the above criterion.

Example 3.2. Let $J=\{0,1,2,4,6,7,9,10\}$.

- $\{0,1,2,4\}$ is a $j$-set because $2-1=1$ and $4-2 \geq 2-0$.

- $\{0,1,2,4,6\}$ is a $j$-set because $6-4 \geq 4-2$.

- $\{0,1,2,4,6,7\}$ is a $j$-set because $7-6=1$.

- $\{0,1,2,4,6,7,9\}$ is not a $j$-set because $7-6=1$ and $9-7<7-4$.

Thus, $J$ is not a $j$-set.

\section{Overpartitions, $j$-sequences and an exact formula for $F(x, y, z)$.}

Instead of counting the $j$-sets directly, we will count $j$-sequences which are in one-toone correspondence with $j$-sets. A $j$-sequence is a sequence of overpartitions which are introduced in [3]. We adopt their definition of overpartition.

Definition 3.3. An overpartition of $n$ is a weakly increasing sequence of natural numbers summing to $n$ in which the first occurrence of a number may be overlined.

The original definition is weakly decreasing instead of weakly increasing.

Example 3.4. The sequence $(2,2,3, \overline{5}, 5,5,7)$ is an overpartition of 29 .

For a $j$-set $J=\left\{a_{1}, a_{2}, \ldots, a_{k}\right\}$, where $a_{1}<a_{2}<\cdots<a_{k}$, define the corresponding $j$-sequence $\phi(J)$ as follows. First we define $d(J)$ and $D(J)$ by

$$
\begin{gathered}
d(J)= \begin{cases}\overline{a_{k}-a_{k-2}}, & \text { if } a_{k-1}=a_{k}-1 \text { and } a_{k-1} \neq 0, \\
a_{k}-a_{k-1}, & \text { otherwise, }\end{cases} \\
D(J)= \begin{cases}J \backslash\left\{a_{k-1}, a_{k}\right\}, & \text { if } a_{k-1}=a_{k}-1 \text { and } a_{k-1} \neq 0, \\
J \backslash\left\{a_{k}\right\}, & \text { otherwise. }\end{cases}
\end{gathered}
$$

Starting with $J_{0}=J$, define $J_{i}$ inductively by $J_{i}=D\left(J_{i-1}\right)$ for $i=1,2, \ldots, s$ until $J_{s}=\{0\}$. Now define the $j$-sequence $\phi(J)$ of $J$ as

$$
\phi(J)=\left(d\left(J_{s-1}\right), d\left(J_{s-2}\right), \ldots, d\left(J_{0}\right)\right) .
$$

The sequence $\phi(J)$ records how $J$ grows from $\{0\}$, and we can easily recover $J$ from $\phi(J)$. 
Example 3.5. Let $J=\{0,1,2,4,6,7,10\}$. Then

$$
\begin{array}{ll}
J_{0}=\{0,1,2,4,6,7,10\}, & d\left(J_{0}\right)=3, \\
J_{1}=\{0,1,2,4,6,7\}, & d\left(J_{1}\right)=\overline{3}, \\
J_{2}=\{0,1,2,4\}, & d\left(J_{2}\right)=2, \\
J_{3}=\{0,1,2\}, & d\left(J_{3}\right)=\overline{2}, \\
J_{4}=\{0\} . &
\end{array}
$$

Thus, $\phi(J)=(\overline{2}, 2, \overline{3}, 3)$.

For a $j$-set $J$, it follows from the criterion of $j$-sets in Theorem 2.4 that $\phi(J)$ satisfies the following conditions:

- The sum of integers in $\phi(J)$ is the maximum of $J$.

- $\phi(J)$ starts with $(1, \overline{2}, \ldots)$ or $(\overline{2}, \ldots)$.

- $\phi(J)$ has a descent only before an occurrence of $\overline{2}$.

- Any occurrence of $\bar{i}$ with $i \geq 3$ is preceded by an integer less than $i$.

- $|J \backslash\{0\}|=|\phi(J)|+$ (the number of overlined parts in $\phi(J)$ ).

By cutting $\phi(J)$ in front of each occurrence of $\overline{2}$, we can regard a $j$-sequence as a sequence of overpartitions starting with $\overline{2}$, with possibly one exception: The first one can be the overpartition consisting of a single 1 .

We put a weight $u^{m} z^{l}$ on an overpartition $\pi$ starting with $\overline{2}$, if it is a partition of $m$ and the number of parts plus the number of overlined parts in $\pi$ is $l$. Note that overlined parts are counted twice in $l$, reflecting the fact that an overlined part corresponds to a pair of elements in a $j$-set as shown in (3). Let $Q(u, z)$ be the generating function of such overpartitions. Then

$$
Q(u, z)=\frac{u^{2} z^{2}}{1-u^{2} z} \prod_{i \geq 3} \frac{1+u^{i} z^{2}}{1-u^{i} z},
$$

and $G(u, z)$ can be expressed as

$$
G(u, z)=\sum_{J \in \mathcal{J}} u^{\max (J)} z^{|J|}=z(1+u z) \frac{Q(u, z)}{1-Q(u, z)} .
$$

So $F(x, y, z)$ has the following expression

$$
F(x, y, z)=\frac{z(1+x y z)}{1-x} \cdot \frac{Q(x y, z)}{1-Q(x y, z)} .
$$

When we substitute $y=z=1$ we get the generating function of $f(n)$, the number of $j$-sets in $S_{n}$.

$$
\begin{aligned}
F(x, 1,1)= & x^{2}+2 x^{3}+4 x^{4}+8 x^{5}+16 x^{6}+30 x^{7}+56 x^{8}+102 x^{9} \\
& +186 x^{10}+336 x^{11}+606 x^{12}+1088 x^{13}+1954 x^{14} \\
& +3502 x^{15}+6278 x^{16}+11246 x^{17}+\cdots .
\end{aligned}
$$


Maple experiments suggest that

$$
\lim _{n \rightarrow \infty} \frac{f(n+1)}{f(n)} \approx 1.791178988 \cdots .
$$

\section{The number of permutations with a given $\mathrm{j}$-set}

For a set $J$, define $p_{n}(J)$ to be the number of $\pi \in S_{n}$ such that $J(\pi)=J$. For $n=$ $2,3,4,5,6$, the number $p_{n}(J)$ of permutations with a given $j$-set is shown in the following table.

\begin{tabular}{|c|c|c|c|c|c|}
\hline$J$ & $S_{2}$ & $S_{3}$ & $S_{4}$ & $S_{5}$ & $S_{6}$ \\
\hline$\{0,1,2,3\}$ & & 4 & 8 & 36 & 204 \\
$\{0,1,2\}$ & 2 & 2 & 6 & 26 & 146 \\
$\{0,1,2,3,4\}$ & & & 8 & 24 & 136 \\
$\{0,1,2,3,4,5\}$ & & & & 16 & 64 \\
$\{0,1,2,4\}$ & & & 2 & 8 & 46 \\
$\{0,1,2,3,4,5,6\}$ & & & & & 32 \\
$\{0,1,2,5\}$ & & & & 4 & 20 \\
$\{0,1,2,3,5\}$ & & & & 4 & 20 \\
$\{0,1,2,3,6\}$ & & & & & 12 \\
$\{0,1,2,6\}$ & & & & & 10 \\
$\{0,1,2,3,4,6\}$ & & & & & 8 \\
$\{0,1,2,4,5\}$ & & & & 2 & 8 \\
$\{0,1,2,5,6\}$ & & & & & 4 \\
$\{0,1,2,3,5,6\}$ & & & & & 4 \\
$\{0,1,2,4,5,6\}$ & & & & & 4 \\
$\{0,1,2,4,6\}$ & & & & & 2 \\
\hline
\end{tabular}

The number of permutations $\pi \in S_{n}$ with $J(\pi)=J$.

Definition 4.1. Let $n, k$ be positive integers with $n \geq k$. Let $\pi=\pi_{1} \pi_{2} \cdots \pi_{n}$ be a permutation in $S_{n}$ and $\sigma=\sigma_{1} \sigma_{2} \cdots \sigma_{k}$ a permutation in $S_{k}$. We say $\pi$ contains $\sigma$ as a subsequence, if $\pi_{i_{1}}=\sigma_{1}, \pi_{i_{2}}=\sigma_{2}, \ldots, \pi_{i_{k}}=\sigma_{k}$ for some $i_{1}<i_{2}<\cdots<i_{k}$.

Recall the matrices $M_{\text {col }}(\pi ; k)$ and $M(\sigma)$. The $(i, j)$-entry of $M_{c o l}(\pi ; k)$ is 1 if and only if $j$ is the $i$-th element from left among $1,2, \ldots, k$ in $\pi$. Thus $M_{c o l}(\pi ; k)=M(\sigma)$ means the arrangements of $1,2, \ldots, k$ in $\pi$ and in $\sigma$ are identical. Hence $\pi$ contains $\sigma$ as a subsequence if and only if $M_{c o l}(\pi ; k)=M(\sigma)$. If both $\pi$ and $\sigma$ are involutions, the following holds.

Proposition 4.2. Let $\pi \in S_{n}$ and $\sigma \in S_{k}$ be involutions. Then $\pi$ contains $\sigma$ as a subsequence if and only if the initial k-pattern of $\pi$ is $\sigma$.

Proof. The permutation $\pi$ contains $\sigma$ as a subsequence if and only if $M_{\text {col }}(\pi ; k)=M(\sigma)$. The initial $k$-pattern of $\pi$ is $\sigma$ if and only if $M_{\text {row }}(\pi ; k)=M(\sigma)$. If $M_{\text {col }}(\pi ; k)=M(\sigma)$, 
then $M_{\text {col }}(\pi ; k)$ is symmetric. Thus by Proposition 1.3 , we have $M_{\text {row }}(\pi ; k)=M_{\text {col }}(\pi ; k)=$ $M(\sigma)$. Similarly, if $M_{\text {row }}(\pi ; k)=M(\sigma)$ then we get $M_{\text {col }}(\pi ; k)=M(\sigma)$. Thus these two conditions are equivalent.

Jaggard [1] found a formula for the number of involutions $\pi \in S_{n}$ containing $\sigma$ as a subsequence.

Theorem 4.3 (Jaggard, 2005). For $\sigma \in S_{k}$ and $n \geq k$, the number of involutions in $S_{n}$ which contain $\sigma$ as a subsequence equals

$$
\sum_{j \in J(\sigma)}\left(\begin{array}{l}
n-k \\
k-j
\end{array}\right) t_{n-2 k+j}
$$

where $t_{m}$ is the number of involutions in $S_{m}$ and $t_{0}=1$.

Inspired by the above theorem, we can go on one step further. Using Proposition 4.2, we can convert Theorem 4.3 to the next lemma.

Lemma 4.4. Let $J$ be a j-set with $\max (J)=k$ and $n$ be an integer with $n>k$. Let $i(J, n)$ be the number of involutions $\pi \in S_{n}$ satisfying $J(\pi) \cap[k]=J$. Then

$$
i(J, n)=p_{k}(J) \sum_{j \in J}\left(\begin{array}{l}
n-k \\
k-j
\end{array}\right) t_{n-2 k+j} .
$$

Proof. Let $\pi \in S_{n}$ be an involution with $J(\pi) \cap[k]=J$. Let $\sigma$ be the initial $k$-pattern of $\pi$. Then $J(\sigma)=J$. We can divide the set of such $\pi$ 's as follows.

$$
\bigcup_{\sigma \in S_{k}, J(\sigma)=J}\left\{\pi \in S_{n}: n \in J(\pi), \text { the initial } k \text {-pattern of } \pi \text { is } \sigma\right\}
$$

Since $\pi$ and $\sigma$ are involutions, by Proposition 4.2, this set is equal to

$$
\bigcup_{\sigma \in S_{k}, J(\sigma)=J}\left\{\pi \in S_{n}: n \in J(\pi), \pi \text { contains } \sigma \text { as a subsequence }\right\} .
$$

Thus by Theorem 4.3, the number of $\pi$ is

$$
\sum_{\sigma \in S_{k}, J(\sigma)=J} \sum_{j \in J(\sigma)}\left(\begin{array}{l}
n-k \\
k-j
\end{array}\right) t_{n-2 k+j}
$$

Since $J(\sigma)=J$ for all $\sigma$ in the summation, we are done.

On the other hand the number $i(J, n)$ of involutions $\pi \in S_{n}$ with $\max (J)=k$ and $J(\pi) \cap[k]=J$ has the following expression:

$$
i(J, n)=\sum_{A \subset\{k+1, k+2, \ldots, n-1\}} p_{n}(J \cup A \cup\{n\})
$$

So we obtain the following proposition. 
Proposition 4.5. Let $J$ be a j-set with $\max (J)=k$. Then for $n>k$,

$$
p_{n}(J \cup\{n\})=p_{k}(J) \sum_{j \in J}\left(\begin{array}{l}
n-k \\
k-j
\end{array}\right) t_{n-2 k+j}-\sum_{A} p_{n}(J \cup A \cup\{n\}),
$$

where the last summation is over all nonempty subsets of $\{k+1, \ldots, n-1\}$.

Proposition 4.5 allows us to compute $p_{n}(J)$ for $j$-sets $J$ with $\max (J)=n$. We can use induction on $n$ and $d=n-\max (J \backslash\{n\})$.

We now turn to computing $p_{n}(J)$ for $j$-sets $J$ with $\max (J)<n$.

Proposition 4.6. Let $J$ be a j-set with $\max (J)=k$. Then for $n>k$,

$$
p_{n}(J)=\frac{n !}{k !} p_{k}(J)-\sum_{i=k+1}^{n} \frac{n !}{i !} p_{i}(J \cup\{i\}) .
$$

Proof. It is equivalent to

$$
\frac{n !}{k !} p_{k}(J)=p_{n}(J)+\sum_{i=k+1}^{n} \frac{n !}{i !} p_{i}(J \cup\{i\}) .
$$

Consider the number of permutations $\pi=\pi_{1} \pi_{2} \cdots \pi_{n} \in S_{n}$ with $J(\pi) \cap[k]=J$. There are $\left(\begin{array}{l}n \\ k\end{array}\right)$ ways of choosing the first $k$ elements for $\pi$. For each choice there are $p_{k}(J)$ allowed arrangements, and the rest can be arranged in $(n-k)$ ! ways. Thus there are $\left(\begin{array}{l}n \\ k\end{array}\right) p_{k}(J)(n-k)$ ! permutations $\pi \in S_{n}$ with $J(\pi) \cap[k]=J$. This is the left hand side. To get the right hand side, we classify such $\pi$ 's according to the minimum of $J(\pi) \backslash[k]$, if nonempty. If $J(\pi) \backslash[k]$ is empty then such $\pi$ 's are counted by $p_{n}(J)$.

Lemma 4.7. Let $J$ be a $j$-set with $\max (J)=k$. Then for $n>k, p_{n}(J \cup\{n\})$ and $p_{n}(J)$ are divisible by $p_{k}(J)$.

Proof. In view of Proposition 4.6, it is sufficient to prove for $p_{n}(J \cup\{n\})$. We induct on $n-k$. If $n-k=1$, then it is derived from Lemma 4.4.

Assume for all $n, k$ with $n-k<r$. For the case $n-k=r$,

$$
p_{n}(J \cup\{n\})=i(J, n)-\sum_{A} p_{n}(J \cup A \cup\{n\}),
$$

where the sum is over all nonempty subsets $A$ of $\{k+1, \ldots, n-1\}$. The number $i(J, n)$ is divisible by $p_{k}(J)$ by Lemma 4.4 . Let $A=\left\{a_{1}, a_{2}, \ldots, a_{s}\right\}$. Then $p_{n}(J \cup A \cup\{n\})=p_{n}(J \cup$ $\left.\left\{a_{1}, a_{2}, \ldots, a_{s}, n\right\}\right)$. From the induction hypothesis we obtain a sequence of divisibility relations,

$$
p_{k}(J)\left|p_{a_{1}}\left(J \cup\left\{a_{1}\right\}\right)\right| \cdots\left|p_{a_{s}}\left(J \cup\left\{a_{1}, a_{2}, \ldots, a_{s}\right\}\right)\right| p_{n}(J \cup A \cup\{n\}) .
$$

Thus $p_{n}(J \cup\{n\})$ is divisible by $p_{k}(J)$. 
Using Lemma 4.7 and a similar argument, we can prove the following divisibility property.

Theorem 4.8. Let $J$, $J^{\prime}$ be $j$-sets such that $\max (J)=k, \max \left(J^{\prime}\right) \leq n$ and $J=J^{\prime} \cap[k]$, then $p_{n}\left(J^{\prime}\right)$ is divisible by $p_{k}(J)$.

Corollary 4.9. For all $J$ and $n, p_{n}(J)$ is even.

Proof. $p_{2}(\{0,1,2\})=2$. Because every $j$-set contains $\{0,1,2\}, p_{n}(J)$ is divisible by $p_{2}(\{0,1,2\})=2$.

\section{References}

[1] Aaron D. Jaggard, Subsequence containment by involutions, Elec. J. Combin. 12 (2005), \#R14.

[2] Brendan D. McKay, Jennifer Morse, and Herbert S. Wilf, The Distributions of the Entries of Young Tableaux, J. Combin. Theory Ser. A 97 (1) (2002), 117-128.

[3] S. Corteel and J. Lovejoy, Overpartitions, Trans. Amer. Math. Soc. 356 (2004), 16231635. 